\title{
SPC-Threshold: Uma Proposta de Limiarização para Filtragem Adaptativa de Sinais
}

F.M. BAYER, ${ }^{1}$ Universidade Federal da Fronteira Sul - Campus Erechim, 99700-000 Erechim, RS, Brasil.

A.J. KOZAKEVICIUS', Departamento de Matemática, Universidade Federal de Santa Maria, 97105-900 Santa Maria, RS, Brasil.

Resumo. Neste trabalho é apresentada uma proposta de limiarização para filtragem adaptativa de sinais por meio do truncamento dos coeficientes wavelets do sinal analisado. O parâmetro de corte para limiarização é estimado por analogia à aplicação dos gráficos de controle, que é uma ferramenta do controle estatístico de processo (SPC - Statistical Process Control). O método proposto, denominado SPC-Threshold, é formulado e para sua validação são realizadas simulações computacionais. Os resultados do SPC-Threshold são comparados com aqueles obtidos com limiares de truncamento já consagrados, como o Universal Threshold, o SURE Threshold e suas variações.

Palavras-chave. SPC-Threshold, Filtragem de Sinais, Wavelets.

\section{Introdução}

A transformada wavelet (TW) tem sido largamente utilizada como ferramenta auxiliar para reconhecimento de padrões, codificação e compressão de dados, eliminação de ruído, decomposição de sinais ou séries temporais, etc [10,16]. Dentre as diversas aplicações da TW, o processo de filtragem de sinais vem recebendo grande atenção por parte da comunidade científica. Algumas aplicações, nas quais técnicas de filtragem utilizando TW já foram testadas e validadas, referem-se à redução de ruído de sinais enviados por satélite [18], filtragem de sinais cardíacos [8, 2], filtragem e compressão de imagens [1], entre outras.

As técnicas de filtragem de sinais envolvendo wavelets têm como marco principal os trabalhos de Donoho e seus colaboradores $[4,5,6]$. A remoção de ruído nem sempre é trivial e imediata, especialmente quando se trata de sinais biomédicos, para os quais a garantia de preservação de informações significativas para análises posteriores é fundamental [11]. Neste trabalho, como uma alternativa às propostas de Donoho, é apresentada uma estratégia de obtenção do limiar de corte para filtragem de sinais por meio da limiarização (truncamento) dos coeficientes wavelets do

\footnotetext{
${ }^{1}$ fabiobayer@gmail.com

2alicek@smail.ufsm.br
}

Recebido em 13 Junho 2009; Aceito em 06 Maio 2010. 
sinal transformado. Como a motivação para a obtenção do limiar de truncamento foi obtida pela determinação dos limites dos gráficos de controle, que são ferramentas do controle estatístico de processo (SPC - Statistical Process Control), a técnica proposta é denominada SPC-Threshold.

O SPC-Threshold é apresentado e comparado por meio de simulações computacionais com os limiares de corte Universal Threshold [4], SURE Threshold [5] e suas derivações. Os resultados dos experimentos computacionais evidenciam aspectos positivos na utilização do limiar de corte proposto neste trabalho. Além das vantagens apresentadas pelo SPC-Threshold frente aos demais limiares quanto à preservação de informações relevantes após o processo de filtragem, os resultados mostram ainda uma maior robustez do método com relação aos diferentes tipos de sinais e níveis de ruído simulados.

O presente artigo está organizado da seguinte maneira. Na Seção 2 é apresentada uma breve introdução sobre a transformada wavelet discreta de uma função e sua série wavelet, considerando-se aqui a família de wavelets ortonormais de Daubechies [3]. Na Seção 3 a operação de truncamento, ou limiarização como denominado em [13], dos coeficientes wavelets para a filtragem de sinais é apresentada, assim como as principais propostas de limiarização conhecidas na literatura. Nesta mesma seção o SPC-Threshold é apresentado em detalhes. Na Seção 4 são apresentados os sinais simulados contendo diferentes níveis de ruído e ainda são apresentados os resultados dos testes computacionais, sob a forma de medidas de qualidade de desempenho da filtragem dos sinais. Por fim, as conclusões encontram-se na Seção 5.

\section{A Transformada Wavelet}

Wavelets são boas alternativas como bases para representação de funções em $L^{2}(\mathbb{R})$, principalmente funções não periódicas para as quais a análise de Fourier deixa de ser a melhor opção. Diferentemente da análise de Fourier, a análise wavelet permite uma fatoração dos dados (discretos ou contínuos) de forma que estes fiquem localizados tanto no tempo quanto nas escalas, o que torna as wavelets uma forte ferramenta para análise de dados não-estacionários, ou com descontinuidades. Nesta seção, a representação de uma função em relação à série wavelet é apresentada, considerando-se a família de wavelets ortonormais de suporte compacto de Daubechies. Para uma abordagem aprofundada sobre representação multiresolução e a construção de wavelets ortonormais recomenda-se a referência clássica de Ingrid Daubechies [3]. Já para uma abordagem mais aplicada e com enfoque em algoritmos das transformadas wavelets, recomenda-se a tese de Olé Nielsen, disponível na web [14].

Em uma análise multiresolução (MRA), dada $f(x) \in L^{2}(\mathbb{R})$ inicialmente discretizada em um nível de resolução, o objetivo principal é representá-la através de suas decomposições em vários níveis de detalhes, além de uma componente mais grosseira. Os detalhes $\left\{d_{j, k}\right\}_{k \in \mathbb{Z}}$ são os coeficientes wavelets de $f(x)$, obtidos através da projeção da $f(x)$ nos espaços $W_{j}$, que são gerados pelas funções wavelets no nível $j,\left\{2^{j / 2} \psi\left(2^{j} x-k\right)\right\}_{k \in \mathbb{Z}}=\left\{\psi_{j, k}(x)\right\}_{k \in \mathbb{Z}}$. Os coeficientes associados à componente mais grosseira são denominados coeficientes de escala $\left\{c_{j, k}\right\}_{k \in \mathbb{Z}}$ 
e são obtidos através da projeção da $f(x)$ no espaço $V_{j}$, gerado pelas funções escala $\left\{2^{j / 2} \phi\left(2^{j} x-k\right)\right\}_{k \in \mathbb{Z}}=\left\{\phi_{j, k}(x)\right\}_{k \in \mathbb{Z}}$. Como $V_{j}=V_{j-1} \oplus W_{j-1}, \forall j$, entre dois níveis consecutivos $P_{V_{j}} f=P_{V_{j-1}} f+P_{W_{j-1}} f$. Com isso, considerando que o nível mais grosseiro seja $j_{0}$, a representação com diversos níveis de fatoração é dada por:

$$
\left(P_{V_{j}} f\right)(x)=\sum_{l=-\infty}^{+\infty} c_{j_{0}, l} \phi_{j_{0}, l}(x)+\sum_{k=j_{0}}^{j-1} \sum_{l=-\infty}^{+\infty} d_{k-1, l} \psi_{k-1, l}(x) .
$$

A ortogonalidade entre as funções $\left\{\phi_{j, k}(x)\right\}_{k \in \mathbb{Z}}$ e $\left\{\psi_{j, k}(x)\right\}_{k \in \mathbb{Z}}$ associada à relação de escala que caracteriza a MRA induz uma relação entre $\left\{c_{j, l}\right\}_{l \in \mathbb{Z}}$ do nível mais fino $j$ com a sequência de coeficientes $\left\{c_{j-1, l}\right\}_{l \in \mathbb{Z}}$ e $\left\{d_{j-1, l}\right\}_{l \in \mathbb{Z}}$, envolvendo os filtros da relação de escala. Isto produz um algoritmo rápido e acurado, denotado algoritmo de Mallat ou piramidal, que define a TW rápida entre dois níveis consecutivos, ou entre uma número maior de níveis intermediários de fatoração [9].

\section{Limiarização de Coeficientes Wavelets}

Quando informações discretas são obtidas através de medições ou manipuladas por aplicativos computacionais e então armazenadas digitalmente, em alguma etapa deste processo as informações podem ser alteradas por diversas formas de interferência, denominadas de ruídos.

As técnicas de limiarização dos coeficientes de uma série wavelet tem como objetivo a redução, ou mesmo eliminação, do ruído presente em um sinal. Estas técnicas estão baseadas na manipulação dos coeficientes wavelet, que podem ter seus valores diminuídos ou anulados nos vários níveis de fatoração gerados pela TW direta. Após esse processo, é aplicada a TW inversa na série wavelet com coeficientes limiarizados [13]. O sinal reconstruído será uma aproximação do sinal sem ruído.

Considera-se o vetor $T$-dimensional $\mathbf{f}=\left(f_{1}, f_{2}, \ldots, f_{T}\right)$, com $T=2^{J_{\max }}$, sendo cada componente uma amostra do sinal sem ruído $f$ em um instante $t_{i}$, ou seja $f_{i}=f\left(t_{i}\right)$. Considera-se ainda o vetor de ruído gaussiano $\epsilon=\left(\epsilon_{1}, \ldots, \epsilon_{T}\right)$, cujas componentes são variáveis aleatórias independentes e identicamente distribuídas com distribuição normal de média zero e variância $\sigma^{2}$, ou seja, $\epsilon_{i} \sim$ i.i.d. $N\left(0, \sigma^{2}\right)$, para $i=1, \ldots, T$. Desta forma, segundo formulado por Donoho, um sinal amostrado $\mathbf{Y}$ pode ser considerado como uma soma de uma componente limpa f e um ruído $\epsilon$, ou seja:

$$
\begin{aligned}
\mathbf{Y} & =\mathbf{f}+\epsilon \\
\left(y_{1}, y_{2}, \ldots, y_{T}\right) & =\left(f_{1}+\epsilon_{1}, f_{2}+\epsilon_{2}, \ldots, f_{T}+\epsilon_{T}\right) .
\end{aligned}
$$

Na prática, a única informação conhecida é o vetor $\mathbf{Y}$, e o objetivo então é, a partir de $\mathbf{Y}$, obter uma estimativa para a componente $\mathbf{f}$, de tal modo que se tenha um risco mínimo. O risco é definido como o valor esperado de um determinada função perda. Neste trabalho, assim como em $[4,5]$, o erro quadrático médio (EQM) é considerado como função perda. Portanto, busca-se uma estimativa $\hat{\mathbf{f}}=\left(\hat{f}_{1}, \hat{f}_{2}, \ldots, \hat{f}_{T}\right)$ de $\mathbf{f}$ que minimize

$$
\mathrm{EQM}=\frac{1}{T} \sum_{i=1}^{T}\left(\hat{f}_{i}-f_{i}\right)^{2}
$$


A estimativa $\hat{\mathbf{f}}$ é obtida através da limiarização dos coeficientes wavelet da série de f. A operação $\operatorname{thr}_{\lambda}(\cdot)$ reduz ou anula os coeficientes $d_{j, l}$ (equação (2.1)) menores que certo escalar $\lambda$. As duas propostas de truncamento mais difundidas [13], denominadas de Hard e Soft Thresholding, são dadas respectivamente por:

$\operatorname{thr}_{\lambda}^{H}\left(d_{j, l}\right)=\left\{\begin{array}{ll}0, & \text { se }\left|d_{j, l}\right| \leq \lambda \\ d_{j, l}, & \text { se }\left|d_{j, l}\right|>\lambda\end{array}, \operatorname{thr}_{\lambda}^{S}\left(d_{j, l}\right)=\left\{\begin{array}{ll}0, & \text { se }\left|d_{j, l}\right| \leq \lambda, \\ \operatorname{sign}\left(d_{j, l}\right)\left(\left|d_{j, l}\right|-\lambda\right), & \text { se }\left|d_{j, l}\right|>\lambda\end{array}\right.\right.$.

Segundo [13], o Soft Thresholding apresenta vieses maiores, enquanto que o Hard Thresholding tem viés menor, porém variância maior. Portanto a escolha entre Hard ou Soft é muitas vezes feita caso a caso, dependendo da aplicação. Dada a função $\operatorname{thr}_{\lambda}(\cdot)$, o procedimento para filtragem do sinal $\mathbf{Y}$, por meio de limiarização dos coeficientes wavelets, segue as seguintes etapas: (a) Aplicação da TW direta no sinal original $\mathbf{Y}: \mathrm{TW}(\mathbf{Y})=\breve{\mathbf{Y}}$; (b) Aplicação da função de truncamento a todos os coeficientes wavelets de $\mathbf{Y}$ obtidos no passo anterior: $\operatorname{thr}_{\lambda}(\breve{\mathbf{Y}})=\widetilde{\mathbf{Y}}$; (c) Aplicação da TW inversa na série de coeficientes wavelets truncados: $\operatorname{TWI}(\widetilde{\mathbf{Y}})=\hat{\mathbf{f}}$. No final do procedimento de filtragem é obtida uma estimativa $\hat{\mathbf{f}}$ do sinal sem ruído $\mathbf{f}$.

Por ser uma transformação linear, temos que $\mathrm{TW}(\mathbf{Y})=\mathrm{TW}(\mathbf{f}+\epsilon)=\mathrm{TW}(\mathbf{f})+$ TW $(\epsilon)$ e, por ser ortogonal, a TW ainda preserva a energia do sinal analisado. Portanto, no caso deste sinal ser um ruido gaussiano, suas propriedades de média zero e variância $\sigma^{2}$ são preservadas após a transformação. Em termos de coeficientes wavelets, esta constatação pode ser expressa por: $d_{j, l}=w_{j, l}+\sigma z_{j, l}$, sendo $d_{j, l}$ os coeficientes wavelet de $\mathbf{Y}$ e $w_{j, l}$, os coeficientes wavelets de $\mathbf{f}$, e ainda $z_{j, l} \sim$ i.i.d. $N(0,1)$. Ou seja, os coeficientes wavelets de uma amostra com ruído podem ser escritos como os coeficientes wavelets sem ruído adicionados a ruído branco [13].

Agora, o problema a ser considerado é a determinação do limiar de corte $\lambda$, cuja escolha é fundamental para a eficiência do processo de filtragem.

\subsection{Determinação do limiar de corte}

O limiar de corte $\lambda$ depende de um parâmetro de dispersão $\sigma$. O parâmetro $\sigma$ pode ser estimado através de duas abordagens: a primeira, global, assume que existe um único parâmetro $\sigma$ para todos os coeficientes wavelet nos diferentes níveis da transformação; a segunda abordagem, por nível, considera que existe um parâmetro $\sigma_{j}$ para cada nível de resolução $j$, implicando em um limiar de corte $\lambda_{j}$ diferente a cada escala $j$. Neste trabalho utiliza-se o desvio padrão amostral dos coeficientes como estimativa de $\sigma$, no entanto, ele pode ser determinado por meio de outros estimadores $[13,6]$. Apresentam-se a seguir as principais formulações para determinação do limiar de corte $\lambda$, assim como o SPC-Threshold proposto neste trabalho.

\subsubsection{Universal Threshold}

Em [4], Donoho e Johnstone propõem um estimador denominado VisuShrink, no qual $\lambda$ é dado por: $\lambda=\sigma \sqrt{2 \log (T)}$, sendo $\sigma$ estimado a partir dos dados, podendo ser uma abordagem por nível com um parâmetro de dispersão para cada nível, ou de forma global, com um único parâmetro de dispersão para todas escalas. 


\subsection{2. $\quad$ SURE Threshold}

O procedimento SureShrink [5] minimiza um estimador não-viesado do risco de Stein (SURE - Stein Unbiased Risk Estimator) a cada nível de resolução $j$ [19]. Este processo caracteriza uma operação adaptativa, uma vez que a cada nível, a determinação do limiar de corte depende dos coeficientes wavelets do sinal. Sendo $\sigma_{j}$ o parâmetro de dispersão e $T_{j}$ o número de coeficientes wavelet do nível $j$, define-se o limiar de corte por:

$$
\lambda_{j}=\underset{0 \leq t \leq \sigma_{j} \sqrt{2 \log \left(T_{j}\right)}}{\operatorname{argmin}} \operatorname{SURE}\left(\mathbf{d}_{j}, t\right),
$$

com

$$
\operatorname{SURE}\left(\mathbf{d}_{j}, t\right)=T_{j}-2 \sum_{l=1}^{T_{j}} I\left(\left|d_{j, l}\right| \leq t\right)+\sum_{l=1}^{T_{j}} \min \left(\left|d_{j, l}\right|, t\right)^{2},
$$

na qual $\sigma_{j}$ é a medida de disperão para cada nível $j$ e $I(x)=\left\{\begin{array}{ll}1, & \text { se } x=\text { verdadeiro } \\ 0, & \text { se } x=\text { falso }\end{array}\right.$.

Contudo, em [8] é proposta uma variação do SURE, assumindo que existe um único parâmetro de dispersão $(\sigma)$, o qual é estimado a partir dos coeficientes wavelet de todos os níveis de resolução. Este limiar será denominado SURE global.

\subsubsection{SPC-Threshold}

O SPC-Threshold foi motivado pela utilização dos gráficos de controle de Shewhart [17], ferramenta do SPC geralmente utilizada para alcançar um estado de controle estatístico e para monitorar um determinado processo, distinguindo causas de variabilidade entre comuns e especiais. As comuns seriam originárias da variabilidade natural do processo, cuja variação possui um comportamento puramente aleatório, indicando que o processo está sob controle. As causas especiais revelam a formação de padrões distintos, acusando que algo incomum está influenciando o processo.

Um diagrama de controle de Shewhart é constituído de um sistema de eixos cartesianos ortogonais: no eixo das abscissas são representados os instantes de registro das informações e no eixo das ordenadas, a medida da variável $x$ correspondente a cada instante. Uma linha central (LC), paralela ao eixo das abscissas, representa o valor médio da variável correspondente ao estado do processo sob controle. Duas linhas paralelas à LC, respectivamente, acima e abaixo desta, são traçadas correspondendo aos limites superior de controle (LSC) e limite inferior de controle (LIC) [12]. Estes limites de controle para o gráfico de Shewhart são dados por:

$$
\mathrm{LSC}=\bar{X}+p S, \quad \mathrm{LC}=\bar{X}, \quad \mathrm{LIC}=\bar{X}-p S,
$$

sendo $\bar{X}$ um estimador da média do processo, $S$ um estimador do desvio padrão $\sigma$ e $p$ é um escalar de valor arbitrário, geralmente considerado igual a 2 ou 3 [12].

Após a determinação dos limites de controle, verifica-se se todas as observações de $x$ estão dentro destes limites. Se uma ou mais estiverem fora, elas devem ser investigadas e, portanto, descartas para o cálculo dos novos limites LSC e LIC. Este processo é repetido até que nenhum ponto fique fora dos limites de controle estipulados em passos anteriores [7]. 
Fazendo uma analogia entre os gráficos de controle e limiarização na filtragem de sinais via TW, deseja-se verificar quais coeficientes wavelets $d_{j, l}$ são puramente aleatórios (ruído) e quais representam alguma causa especial de comportamento (neste caso, o comportamento determinado pelo sinal de interesse). Sendo assim, considerando o vetor $\mathbf{d}_{j}$ com os $T_{j}$ coeficientes wavelets da escala $j$, o limiar de corte $\lambda_{j}$ do SPC-Threshold adaptativo é estabelecido através das seguintes etapas:

(a) Obter uma estimativa de $\sigma_{j}$ por meio de $S_{j}=\sqrt{\frac{1}{T_{j}-1} \sum_{l=1}^{T_{j}}\left(d_{j, l}-\bar{d}_{j}\right)^{2}}$, sendo $\bar{d}_{j}=\frac{1}{T_{j}} \sum_{l=1}^{T} d_{j, l}$ a média aritmética dos coeficientes wavelets do nível $j ;$

(b) Se $\left|d_{j, l}\right|>p S_{j}$, então este coeficiente é descartado do vetor $\mathbf{d}_{j}$ e retorna-se a etapa (a) para obtenção de um novo valor $S_{j}$. Se $\left|d_{j, l}\right|<p S_{j}, \forall l$, então prossegue-se a etapa (c);

(c) O valor do limiar de corte é dado por $\lambda_{j}=p S_{j}$.

Além dessa abordagem adaptativa, na qual um $\lambda_{j}$ para cada nível de resolução $j$ é determinado, pode-se ainda obter uma abordagem global. Para o SPC-Threshold global, o vetor $\mathbf{d}_{j}$ é construído agora com todos os coeficientes wavelets $d_{j, l}$ de todos os níveis de fatoração da TW. Para a determinação do limiar, as mesmas etapas (a), (b) e (c) são realizadas.

\section{Estudo de Simulação}

Para as simulações apresentadas nessa seção, foram aplicadas TW com fatoração de 1 a 7 níveis, sendo que para cada um dos níveis foram consideradas wavelets de Daubechies com momentos nulos variando de 1 a 7 , totalizando 49 variações. Ainda, para cada uma dessas 49 variações foram consideradas 100 réplicas de Monte Carlo, obtendo um total de 4900 simulações para cada sinal. A implementação computacional foi realizada utilizando-se o software livre $\mathrm{R}$ versão 2.6.0 [15]. Os sinais simulados possuem $T=1024=2^{10}$ observações igualmente espaçadas no seu nível mais fino de resolução e são provenientes de três diferentes funções, a saber:

(i) Função Doppler, utilizada em $[4,13]$ para testes e ilustração de técnicas de limiarização, cuja definição é: $f\left(t_{i}\right)=\sqrt{\left(t_{i}\left(1-t_{i}\right)\right)} \operatorname{sen}\left(2,1 \pi /\left(t_{i}+0,05\right)\right)$.

(ii) ECG Simulado, que é um vetor numérico que simula um eletrocardiograma de uma pessoa com batimentos cardíacos normais.

(iii) Função Wave, que é uma função periódica e suave definida da seguinte maneira: $f\left(t_{i}\right)=10 \operatorname{sen}\left(t_{i} / 50\right)+\cos \left(t_{i} / 100\right)$.

Em cada um dos três sinais são somados três diferentes níveis de ruído gaussiano, totalizando nove sinais ruidosos distintos, apresentados na Figura 1. O objetivo da simulação computacional é comparar o desempenho das técnicas de limiarização quando aplicadas à filtragem de sinais, para a qual o esquema de limiarização 

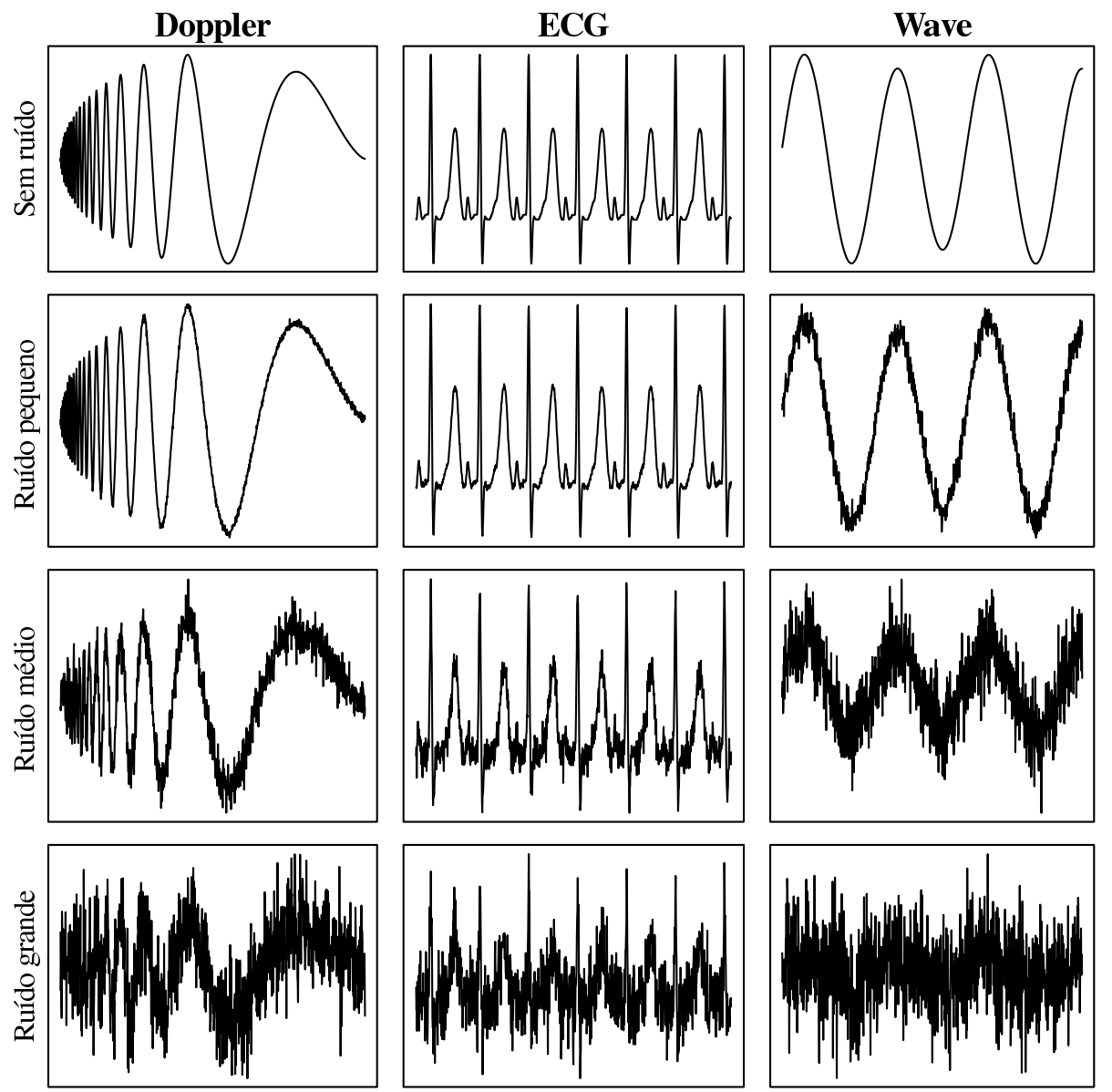

Figura 1: Função Doppler, sinal ECG e função Wave com diferentes níveis de ruído.

escolhido foi o Soft Thresholding. Cada um dos limiares apresentados na Seção 3 possuem duas variações de acordo com a suposição feita sobre o parâmetro de dispersão $\sigma$. Com isso, tem-se um total de seis limiares de corte analisados, que são:

T1 - SPC-Threshold global, assumindo um único $\sigma$ para todos os níveis;

T2 - SPC-Threshold por nível, com $\sigma_{j}$ para cada nível $j$;

T3 - Universal Threshold global, assumindo um único $\sigma$ para todos os níveis;

T4 - Universal Threshold por nível, $\operatorname{com} \sigma_{j}$ para cada nível $j$;

T5 - SURE Threshold global, assumindo um único $\sigma$ para todos os níveis;

T6 - SURE Threshold, com $\sigma_{j}$ para cada nível $j$.

Nas simulações apresentadas nesta seção o parâmetro $p$ do SPC-Threshold é fixado em $p=2$. Para este caso, tem-se uma probabilidade igual a 0,0456 de um 
ponto ser erroneamente considerado sinal de interesse quando na verdade é ruído. Isso deve-se ao fato da probabilidade de uma variável aleatória com distribuição normal e média zero ter uma ocorrência entre menos dois desvios padrão e mais dois desvios padrão ser igual a 0,9544. Além disso, em resultados computacionais preliminares, valores de $p$ próximos a 2 obtiveram os melhores resultados em termos de EQM. Como forma de avaliar qualitativamente a filtragem por meio do SPCThreshold com diferentes valores de $p$, apresenta-se a Figura 2.

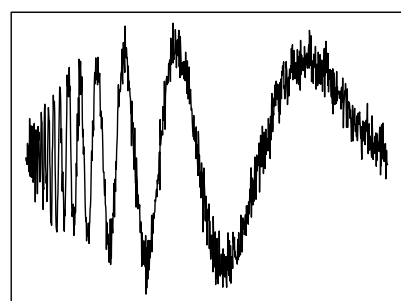

(a) $p=1,5$

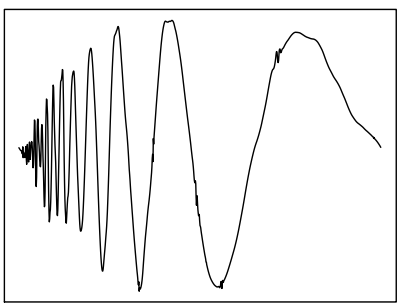

(d) $p=2,25$

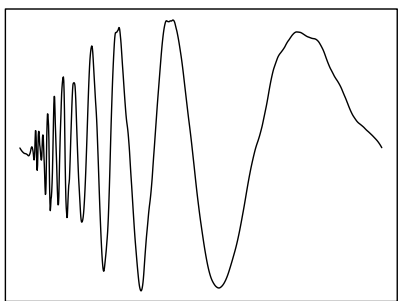

(g) $p=3,25$

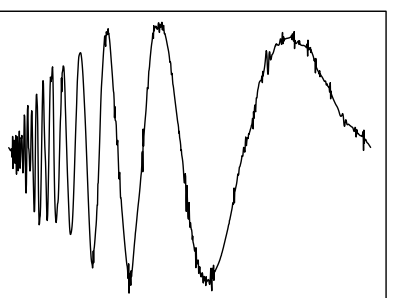

(b) $p=1,75$

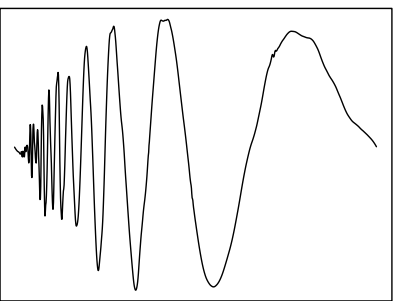

(e) $p=2,5$

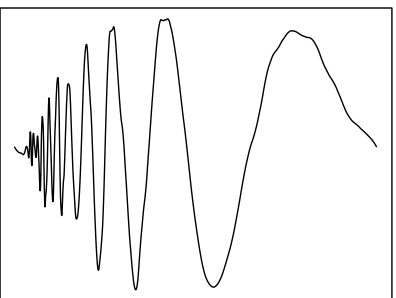

(h) $p=3,5$

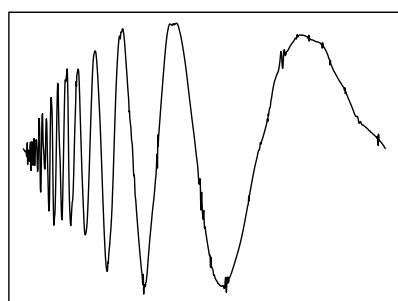

(c) $p=2$

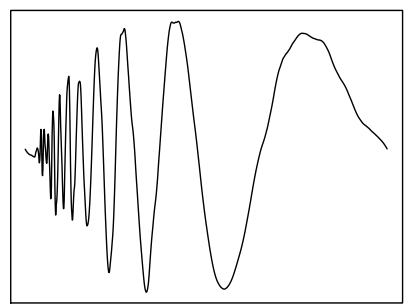

(f) $p=3$

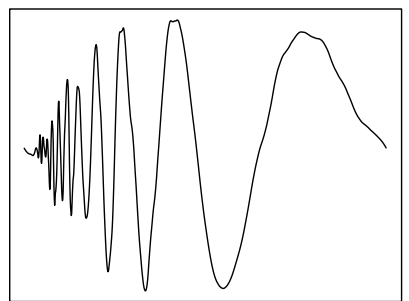

(i) $p=3,75$

Figura 2: Avaliação qualitativa da filtragem via SPC-Threshold com diferentes valores de $p$.

A Figura 2 evidencia que a medida que o valor de $p$ cresce o sinal se torna visualmente mais limpo. No entanto, apesar de ter resultados visuais melhores com valores de $p$ maiores, com valores iguais a $p=2$ e $p=2,25$ os resultados em termos da medida EQM são superiores.

Para conduzir uma análise quantitativa do desempenho de cada uma das técnicas de filtragem é necessário utilizar critérios de comparação. Para tanto, foram selecionadas as medidas de qualidade EQM, conforme definido na equação 3.1, e o 
erro médio absoluto percentual (MAPE) que é definido por:

$$
\text { MAPE }=\frac{1}{T} \sum_{i=1}^{T}\left|\frac{f_{i}-\hat{f}_{i}}{f_{i}}\right| .
$$

Os valores médios das medidas EQM e MAPE para as 4900 realizações de cada sinal estão apresentados nas Tabelas 1 e 2, respectivamente. Nestas tabelas destacamse em negrito os melhores resultados para cada sinal, de acordo com a medida de qualidade considerada.

Tabela 1: Valores médios do EQM da simulação computacional.

\begin{tabular}{|c|c|c|c|c|c|c|c|}
\hline Sinal & Ruído $N(0, \sigma)$ & T1 & T2 & T3 & T4 & T5 & T6 \\
\hline \hline \multirow{3}{*}{ Doppler } & Grande $\sigma=0,271$ & 0,0165 & 0,0192 & 0,0178 & 0,0217 & $\mathbf{0 , 0 1 5 9}$ & 0,0174 \\
\cline { 2 - 8 } & Médio $\sigma=0,072$ & $\mathbf{0 , 0 0 1 6}$ & 0,0042 & 0,0053 & 0,0118 & 0,0040 & 0,0074 \\
\cline { 2 - 8 } & Pequeno $\sigma=0,009$ & $\mathbf{0 , 0 0 0 1}$ & 0,0025 & 0,0040 & 0,0109 & 0,0028 & 0,0064 \\
\hline \multirow{3}{*}{ ECG } & Grande $\sigma=23$ & $\mathbf{1 5 8 , 6 0}$ & 250,68 & 299,21 & 342,49 & 528,97 & 528,97 \\
\cline { 2 - 8 } & Médio $\sigma=5,7$ & $\mathbf{1 5 , 6 2 4}$ & 125,34 & 173,35 & 270,08 & 32,153 & 31,596 \\
\cline { 2 - 8 } & Pequeno $\sigma=0,82$ & 0,8138 & 113,62 & 162,59 & 264,62 & 0,4340 & $\mathbf{0 , 4 3 1 6}$ \\
\hline \multirow{3}{*}{ Wave } & Grande $\sigma=20$ & 75,545 & 76,525 & $\mathbf{5 8 , 6 8 0}$ & 58,727 & 399,71 & 399,71 \\
\cline { 2 - 8 } & Médio $\sigma=5,4$ & 5,7909 & 7,3531 & $\mathbf{5 , 1 8 1 2}$ & 6,1410 & 28,011 & 28,025 \\
\cline { 2 - 8 } & Pequeno $\sigma=0,8$ & 0,1637 & 1,9595 & 0,6286 & 2,0653 & 0,1357 & $\mathbf{0 , 1 3 5 6}$ \\
\hline
\end{tabular}

Tabela 2: Valores médios do MAPE da simulação computacional.

\begin{tabular}{|c|c|c|c|c|c|c|c|}
\hline Sinal & Ruído $N(0, \sigma)$ & T1 & T2 & T3 & T4 & T5 & T6 \\
\hline \hline \multirow{3}{*}{ Doppler } & Grande $\sigma=0,271$ & 3,3740 & 3,4593 & 2,9826 & 3,0202 & $\mathbf{2 , 9 5 7 8}$ & 2,9871 \\
\cline { 2 - 8 } & Médio $\sigma=0,072$ & $\mathbf{1 , 0 3 0 5}$ & 1,2195 & 1,1794 & 1,2732 & 1,1295 & 1,2406 \\
\cline { 2 - 8 } & Pequeno $\sigma=0,009$ & $\mathbf{0 , 2 0 5 2}$ & 0,5094 & 0,6419 & 0,7578 & 0,5936 & 0,7279 \\
\hline \multirow{3}{*}{ ECG } & Grande $\sigma=23$ & $\mathbf{3 , 8 1 9 9}$ & 4,7815 & 4,4049 & 4,6275 & 8,1356 & 8,1356 \\
\cline { 2 - 8 } & Médio $\sigma=5,7$ & $\mathbf{1 , 1 7 7 1}$ & 2,9887 & 2,6566 & 3,1618 & 2,0035 & 1,9841 \\
\cline { 2 - 8 } & Pequeno $\sigma=0,82$ & 0,2657 & 2,5526 & 2,2844 & 2,8227 & $\mathbf{0 , 2 0 7 8}$ & 0,2081 \\
\hline \multirow{3}{*}{ Wave } & Grande $\sigma=20$ & 2,9045 & 2,9352 & $\mathbf{2 , 4 6 1 5}$ & 2,4635 & 7,5122 & 7,5122 \\
\cline { 2 - 8 } & Médio $\sigma=5,4$ & 0,8239 & 0,9368 & $\mathbf{0 , 7 7 1 9}$ & 0,8331 & 1,9914 & 1,9918 \\
\cline { 2 - 8 } & Pequeno $\sigma=0,8$ & 0,1418 & 0,3378 & 0,2191 & 0,3378 & $\mathbf{0 , 1 2 1 9}$ & 0,1219 \\
\hline
\end{tabular}

As Tabelas 1 e 2 mostram que, em geral, o SPC-Threshold global obteve os melhores resultados na filtragem dos sinais simulados. Além disso, o SPC-Threshold global mostrou-se bastante robusto na presença de sinais com diferentes características e também sob níveis distintos de ruído. Os valores obtidos pelo SPC-Threshold quando não alcançaram os menores valores para as medidas EQM e MAPE estiveram bastante próximos dos menores valores alcançados.

O bom desempenho diante de diferentes sinais não é mantido nas demais técnicas de limiarização, como evidenciado nas Tabelas 1 e 2. De fato, apesar do SURE Threshold obter os menores EQM em dois casos, ele apresenta o pior resultado nos sinais ECG com grande ruído e função Wave com grande e médio ruído. O mesmo observa-se com o Universal Threshold global, que obteve as menores medidas EQM e MAPE para a função Wave com ruídos grande e médio, mas em todos os outros casos obteve resultados inferiores ao SPC-Threshold global. 
Para uma avaliação do tempo computacional são apresentados na Tabela 3 os tempos despendidos no experimento de simulação utilizando cada um dos limiares investigados. A simulação foi desenvolvida em um computador com processador AMD Athlon X2 $2800 \mathrm{Mhz}, 3$ GB de memória RAM e sistema operacional Linux Ubuntu 9.10. Observa-se que o tempo computacional do limiar proposto é competitivo com o Universal Threshold, apresentando-se computacionalmente mais eficiente do que o SURE Threshold.

Tabela 3: Tempo computacional do estudo de simulação para os diferentes limiares.

\begin{tabular}{|c|c|c|c|c|c|c|}
\hline & T1 & T2 & T3 & T4 & T5 & T6 \\
\hline Tempo em horas & 3,73 & 4,13 & 3,62 & 3,64 & 12,42 & 12,83 \\
\hline
\end{tabular}

Como forma de avaliar qualitativamente os limiares de corte comparados nas tabelas, são apresentados na Figura 3 as filtragens envolvendo cada um dos limiares analisados para a função Doppler com um nível médio de ruído gaussiano. Pode-se observar, comparando os gráficos da Figura 3 com o sinal sem ruído apresentado na Figura 1, que o SPC-Threshold preserva o sinal original da função Doppler, o que não ocorre da mesma forma para os demais limiares considerados. Percebe-se ainda que os demais limiares tendem a eliminar uma maior parte do sinal, degradando informações relevantes no sinal de interesse. Através da Figura 3 percebe-se também que os limiares com parâmetro de dispersão global tendem a ter melhores resultados visuais.

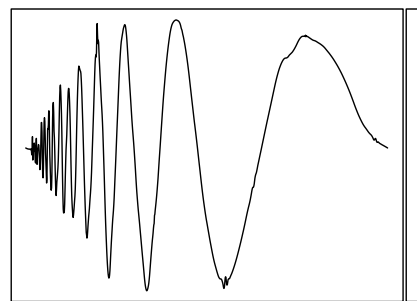

(a) SPC-Threshold Global

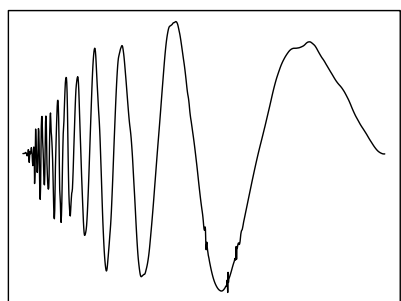

(d) SPC-Threshold por nível

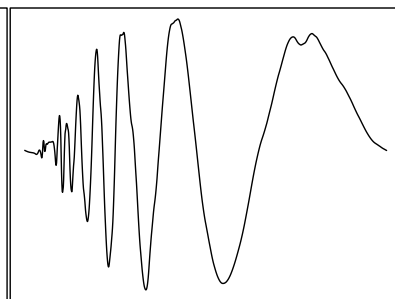

(b) Universal Global

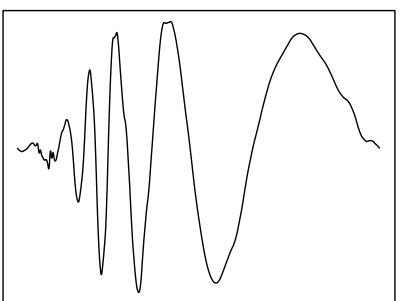

(e) Universal por nível

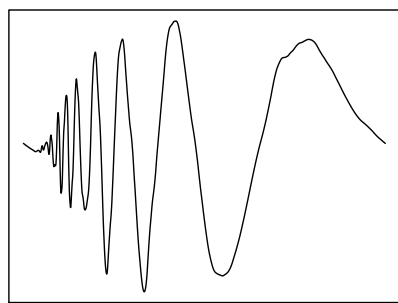

(c) SURE Global

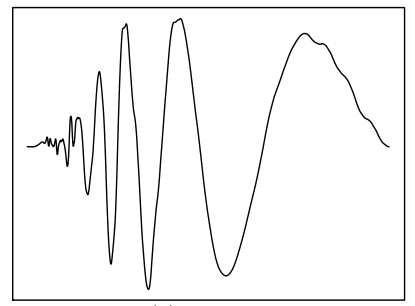

(f) SURE

Figura 3: Filtragem da Função Doppler mais ruído gaussiano $(\sigma=0,072)$, com $T=1024$, Soft Thresholding, 5 momentos nulos e 5 níveis na análise multiresolução. 


\title{
5. Conclusão
}

Este trabalho apresentou uma nova proposta de limiarização para filtragem de sinais, por meio da operação de Soft Thresholding aplicada aos coeficientes wavelets. O novo limiar foi denominado SPC-Threshold pela analogia do procedimento de obtenção do limiar de corte à determinação dos limites de controle nos gráficos de controle. O SPC-Threshold foi comparado computacionalmente com outras técnicas de limiarização já conhecidas e bastante difundidas na literatura. Para a simulação computacional foram utilizadas diferentes funções, assim como diferentes níveis de ruído gaussiano foram agregados a estas funções com o objetivo de avaliar a robustez do SPC-Threshold. Os resultados da comparação das técnicas de limiarização indicam um melhor desempenho do SPC-Threshold e evidenciam sua robustez, uma vez que ele obteve bons resultados em todos os sinais simulados, apresentando uma regularidade de desempenho não observada para os demais limiares analisados.

\begin{abstract}
This work presents an adaptive filter based on thresholding and shrinking wavelet coefficients from the signal wavelet representation. The threshold is estimated by analogy to the application of the control charts, which is a tool of the Statistical Process Control (SPC). The proposed method, called SPC-Threshold, is formulated and computational simulations are used for its validation. The results of the SPC-Threshold are compared with wavelet thresholds already established, such as Universal Threshold, the SURE Threshold and their variations.
\end{abstract}

\section{Referências}

[1] G. Chang, B. Yu, M. Vetterli, Adaptive wavelet thresholding for image denoising and compression, IEEE Transactions on Image Processing, 9, No. 9 (2000), 1532-1546.

[2] G.D. Clifford, A. Shoeb, P.E. Mcsharry, B.A. Janz, Model-based filtering, compression and classification of the ECG, Inter. J. of Bioelectromagnetism, 7, No. 1 (2005), 158-161.

[3] I. Daubechies, Orthonormal bases of compactly supported wavelets, Comm. Pure Applied Mathematics, 41 (1988), 909-996.

[4] D.L. Donoho, I.M. Johnstone, Ideal spatial adaptation via wavelet shrinkage, Biometrika, 81 (1994), 425-455.

[5] D.L. Donoho, I.M. Johnstone, Adapting to unknow smoothing via wavelet shrinkage, J. American Statistical Association, 90 (1995), 1200-1224.

[6] D.L. Donoho, I.M. Johnstone, G. Kerkyacharian, D. Picard, Wavelet shrinkage: assimptopia?, J. Royal Statistical Society serie B, 57 (1995), 301-369.

[7] L.A. Jones, C.W. Champ, Phase I control charts for times between events, Quality and Reliability Engineering International, 18 (2002), 479-488. 
[8] A. Kozakevicius, C.R. Rodrigues, R.C. Nunes, R. Guerra-Filho, Adaptive ECG filtering and QRS detection using orthogonal wavelet transform, In IASTED Inter. Conference on BioMedical Engineering, Innsbruck, ACTAPress, 2005.

[9] S.G. Mallat, A theory for multiresolution signal decomposition, the wavelet representation, IEEE Transactions on Pattern Analysis and Machine Intelligence, 11 (1989), 574-693.

[10] S.G. Mallat, "A Wavelet Tour of Signal Processing", Los Angeles, Academic Press-Elsevier, 1998.

[11] F. Mochimaru, Y. Fujimoto, Y. Ishikawa, Detecting the Fetal Eletrocardiogram by Wavelet Theory-Based Methods, Progress in Biomedical Reserch, 7, No. 3 (2002), 185-193.

[12] D.C. Montgomery, "Introduction to Statistical Quality Control", 3.ed., John Wiley e Sons, New York, 1997.

[13] P.A. Morettin, "Ondas e Ondaletas - Da Análise de Fourier à Análise de Ondaletas", Editora da USP, São Paulo, 1999.

[14] O.M. Nielsen, "Wavelets in Scientific Computing", Ph.D. Thesis, Technical University of Denmark, 1998.

[15] R Development Core Team R. A language and environment for statistical computing. R Foundation for Statistical Computing, Vienna, Austria. ISBN 3-900051-07-0, URL http://www.R-project.org, 2007.

[16] Y. Sheng, "Wavelet Transform" in The Transforms and Applications Handbook", (A.D. Poularikas, ed.), 1.ed., Boca Raton, CRC Press and IEEE Press, (1996), 747-827.

[17] W.A. Shewhart, "Economic Control of Quality of Manufactured Product", New York, MacMillan, 1931.

[18] E.M. Souza, A. Pagamisse, M. Meneguette Jr., J.F.G. Monico, Processamento de sinais com wavelets: redução do efeito do multicaminho do sinal GPS, TEMA Tend. Mat. Apl. Comput., 6, No. 1 (2005), 177-186.

[19] C. Stein, Estimation of the mean of a multivariate normal distribution, Annals of Statistics, 9 (1981), 1135-1151. 